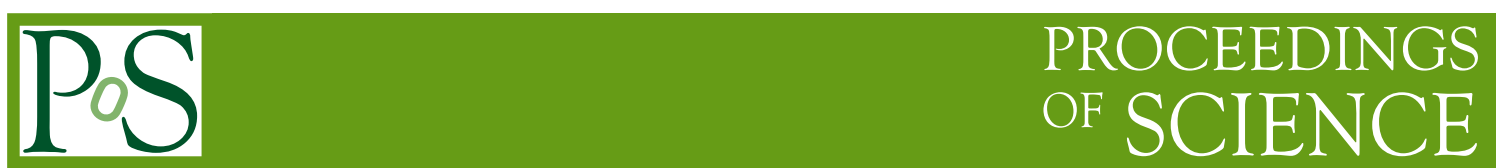

\title{
Recent Results from the MINOS Experiment
}

\author{
Justin J. Evans* for the MINOS Collaboration \\ Department of Physics \& Astronomy, University College London, Gower Street, London, \\ WC1E 6BT, United Kingdom \\ E-mail: evansj@hep.ucl.ac.uk
}

The MINOS experiment utilizes the NuMI beam of muon neutrinos to study the phenomenon of neutrino oscillations. Neutrinos are sent over a baseline of $735 \mathrm{~km}$, with a detector near the production point at Fermilab and one at the Soudan underground laboratory in northern Minnesota. By measuring the disappearance characteristic of oscillations, MINOS has made the best measurement of the atmospheric neutrino mass splitting to date. By looking for electron neutrino appearance, a limit has been placed on the the mixing angle $\sin ^{2}\left(2 \theta_{13}\right)$ of $<0.29$ at $90 \%$ c.l. (for $\delta_{C P}=0$ and normal mass hierarchy). A study of neutral current interactions has allowed limits to be placed on the existence of additional, sterile neutrino flavours beyond the three of the standard oscillation model. Using the 7\% muon antineutrino component of the beam, the first direct observation of muon antineutrinos in a long baseline experiment has been made, along with direct limits on the antineutrino oscillation parameters. In September 2009, the current in the NuMI focusing horns will be reversed to begin running with a dedicated antineutrino beam. This will allow the first precision measurements of the atmospheric-regime antineutrino oscillation parameters, an important test of CPT-invariance in the neutrino sector.

European Physical Society Europhysics Conference on High Energy Physics, EPS-HEP 2009,

July 16 - 222009

Krakow, Poland

${ }^{*}$ Speaker. 


\section{The MINOS Experiment}

MINOS is a long baseline neutrino oscillation experiment. It utilizes the NuMI beam of predominantly muon neutrinos, produced at Fermilab from $120 \mathrm{GeV}$ protons incident on a segmented graphite target. A near detector placed $1 \mathrm{~km}$ from the target measures the neutrino energy spectrum before oscillations have occurred. The far detector, $735 \mathrm{~km}$ from the target, in the Soudan mine, Minnesota, again measures the energy spectrum and a comparison of the two spectra will show any deficits or excesses resulting from oscillations. This two-detector arrangement allows many sources of systematic uncertainty, such as in the neutrino flux or cross sections, to cancel.

The detectors are tracking, sampling calorimeters consisting of alternate planes of steel and scintillator strips [1]. $\mathrm{A} \sim 1.3 \mathrm{~T}$ magnetic field allows particle charge separation and momentum measurement.

\section{2. $v_{\mu}$ Oscillation Analysis}

Figure 1 shows the charged current (CC) $v_{\mu}$ energy spectrum measured at the far detector: 848 events are observed. Also shown is the prediction obtained from the near detector in the case of no oscillations: $1065 \pm 60$ events are expected, showing a clear energy-dependent deficit attributable to oscillations. The data are fit to the oscillation hypothesis in the two-flavour approximation

$$
P\left(v_{\mu} \rightarrow v_{\mu}\right)=1-\sin ^{2}\left(2 \theta_{23}\right) \sin ^{2}\left(\frac{1.27 \Delta m_{\mathrm{atm}}^{2} L}{E}\right)
$$

for the atmospheric neutrino mass splitting $\Delta m_{\text {atm }}^{2}\left[\mathrm{eV}^{2}\right]$ and mixing angle $\theta_{23}$, where $E[\mathrm{GeV}]$ is the neutrino energy and $L[\mathrm{~km}]$ the baseline. This yields the best fit prediction shown in figure 1 . The measured oscillation parameters are $\Delta m_{\mathrm{atm}}^{2}=2.43 \pm 0.13 \times 10^{-3} \mathrm{eV}^{2}, \sin ^{2}\left(2 \theta_{23}\right)>0.9$ (90\% c.l.) [2]. The oscillation parameter space allowed by the data is shown in figure 2 and compared to results from other experiments.

The data were also fit for the alternative disappearance models of pure neutrino decay [3] and

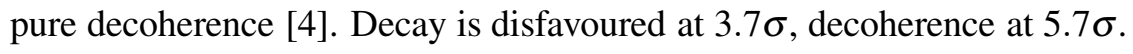

\section{Muon Antineutrinos}

The NuMI beam contains a 7\% background of muon antineutrinos. In the CC interaction $\bar{v}_{\mu}+$ $X \rightarrow \mu^{+}+X^{\prime}$ a positive muon is produced. The magnetized detectors allow this to be distinguished from the negative muon produced in CC- $v_{\mu}$ interactions. The resulting sample of CC- $\bar{v}_{\mu}$ events, with a purity of $97 \%$, allows the same oscillation analysis to be performed as for the CC- $v_{\mu}$ events. However the statistics are considerably lower, and in addition the peak of the $\bar{v}_{\mu}$ spectrum is at $\sim 8 \mathrm{GeV}$, away from the expected signal region (for the $v_{\mu}$ oscillation parameters, the oscillation maximum is at $\sim 2 \mathrm{GeV}$ ); the $\bar{v}_{\mu}$ result will therefore have much lower precision.

For equal $v_{\mu}$ and $\bar{v}_{\mu}$ oscillation parameters, the far detector prediction is shown in figure 3; $58.3 \pm 7.6$ (stat.) \pm 3.6 (syst.) events are expected (for no oscillations, the expectation is $64.6 \pm$ 8.0(stat.) \pm 3.9 (syst.)). The far detector data is also shown in figure 3: 42 events are observed. The deficit in the event rate, visible over all energies, is at the level of $1.9 \sigma$. Using equation 2 to fit for 


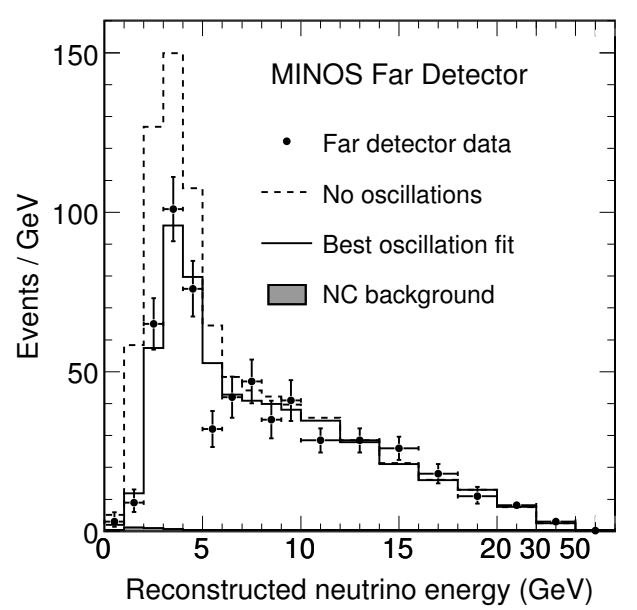

Figure 1: The reconstructed energy spectrum of selected charged current $v_{\mu}$ events at the far detector, also showing the prediction in the case of no disappearance and the best fit to oscillations.

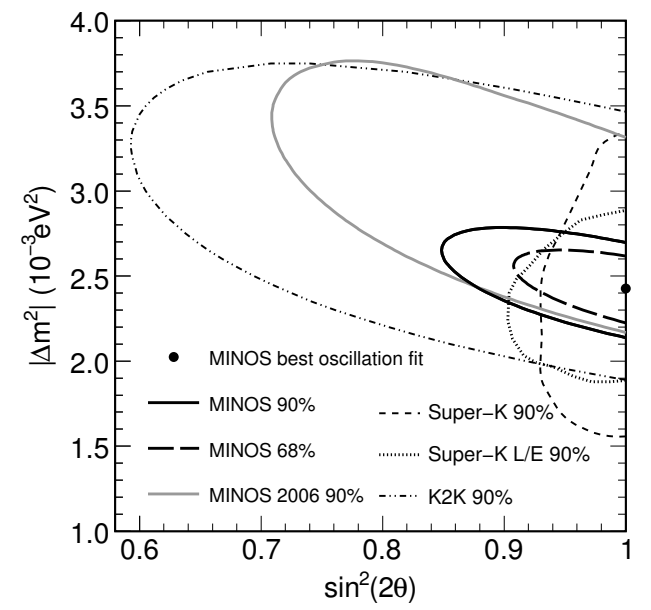

Figure 2: Regions of oscillation parameter space allowed by the MINOS data, compared to other experimental results.

the oscillation parameters an allowed region is obtained as shown in figure 4; this is consistent with the $v_{\mu}$ oscillation parameters at the $90 \%$ c.l. In the figure, the limits obtained are compared with those from a global fit prior to MINOS data [5]; the MINOS data rules out, at high significance, a region of previously allowed parameter space. At maximal mixing MINOS disfavours, at $90 \%$ c.l., $\Delta \bar{m}_{\mathrm{atm}}^{2}<2.0 \times 10^{-3} \mathrm{eV}^{2}$ and $5.0 \times 10^{-3} \mathrm{eV}^{2}<\Delta \bar{m}_{\mathrm{atm}}^{2}<80 \times 10^{-3} \mathrm{eV}^{2}$.

MINOS can also place limits on the process $v_{\mu} \rightarrow \bar{v}_{\mu}$ : a number of $v_{\mu}$ have been seen to disappear, and are assumed to be oscillating predominantly to $v_{\tau}$; but this has not been directly observed. If some were oscillating to $\bar{v}_{\mu}$ this would show up as an excess in the $\bar{v}_{\mu}$ sample. The data of figure 4 can be fit to a simple parameterization assuming a fraction $\alpha$ of the missing $v_{\mu}$ undergo the transition. A limit is obtained of $\alpha<0.026$ (90\% c.l.).

\section{Neutral Current Interactions}

The neutral current (NC) interaction rate is unaffected by oscillations between active neutrino flavours. A deficit in the neutral current event rate at the far detector would be evidence of additional, sterile neutrino flavours. NC events are characterised by a hadronic shower with no muon track. They are selected by looking for events shorter than 60 detector planes, then for events with no reconstructed track, or a track which extends no more than five planes from the hadronic shower [6].

Comparing the data with the expectation calculated from the near detector, no deficit is seen. Quantifying the ratio of observed to expected NC events as $R$, a value $R=1.04 \pm 0.08$ (stat.) \pm 0.07 (syst.) is observed (integrated over all energies), which is consistent with no mixing to sterile 


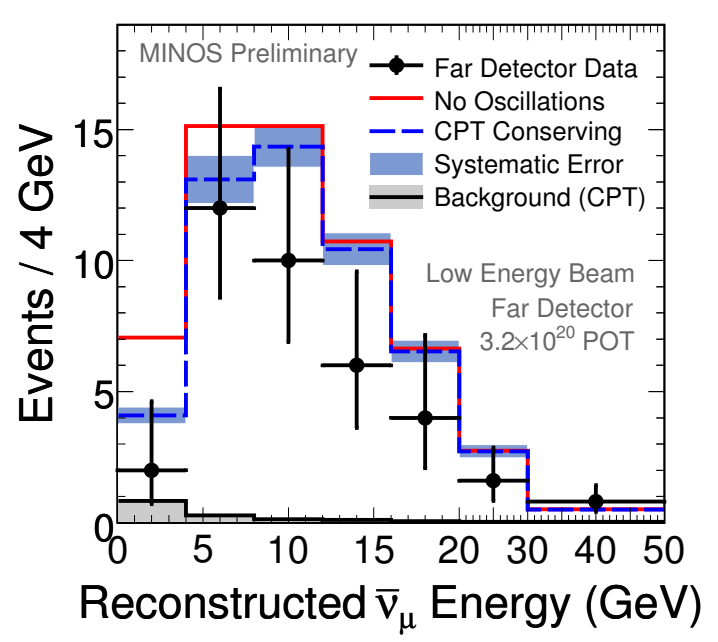

Figure 3: The $\bar{v}_{\mu}$-CC energy spectrum observed in the far detector and the prediction with no oscillations (red) and with oscillation parameters of $\Delta \bar{m}_{\text {atm }}^{2}=\Delta m_{\text {atm }}^{2}=2.43 \times 10^{-3} \mathrm{eV}^{2}, \sin ^{2}\left(2 \bar{\theta}_{23}\right)=$ $\sin ^{2}\left(2 \theta_{23}\right)=1.0$. The blue band encompasses the total systematic uncertainty on the prediction. The estimated background in the CPT-conserving prediction is indicated.

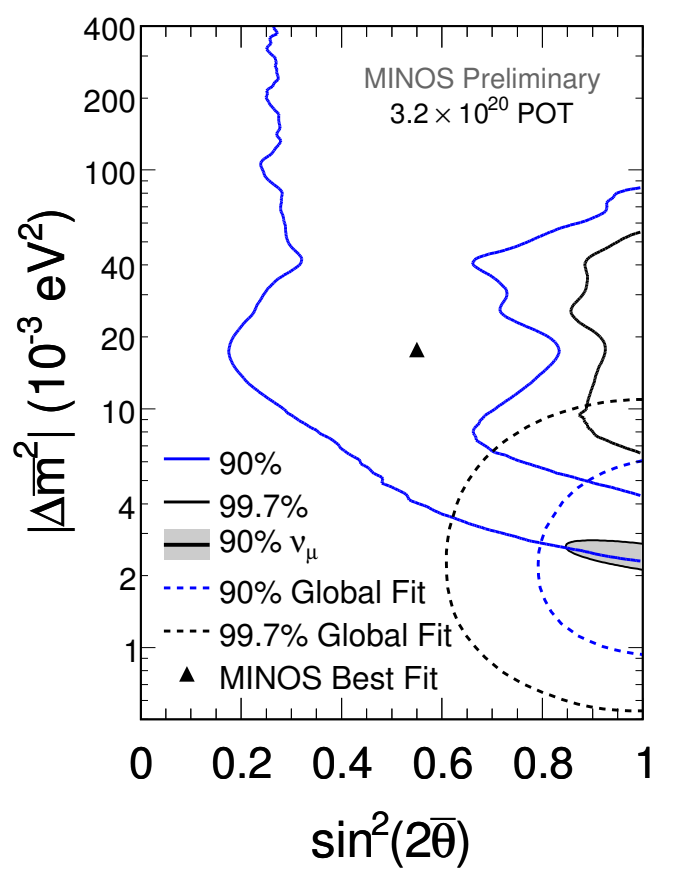

Figure 4: Allowed regions for $\bar{v}_{\mu}$ oscillation parameters from a fit to the data in figure 3. Limits from a global fit [5] and the MINOS allowed region for $v_{\mu}$ oscillations [2] are also shown.

neutrinos. In the case of $\theta_{13}$ at the Chooz limit [7], $R$ is decreased to $R=0.94 \pm 0.08$ (stat.) \pm 0.07 (syst.) (as the resulting additional CC- $v_{e}$ interactions will be included in the NC sample).

\section{5. $v_{e}$ Appearance Search}

If $\theta_{13}$ is non-zero, this will cause oscillations through the appearance channel $v_{\mu} \rightarrow v_{e}$. These CC- $v_{e}$ events are selected by looking for events with compact electromagnetic showers with a signal efficiency of $41 \%$.

At the near detector, discrepancies are seen between the data and simulated $v_{e}$-CC-like spectra at the $20 \%$ level; these spectra consist predominantly of CC- $v_{\mu}$ and NC backgrounds. To correct the simulation for these discrepancies, data is used with the NuMI focusing horns switched off. This significantly changes the contributions of the two background components in a well-modeled way, allowing a measure of their relative contributions which is used to correct the far detector expectation. The final systematic uncertainty on the predicted far detector event rate is $7.3 \%$ with a statistical uncertainty of $19 \%$.

At the far detector, 37 events are seen for an expected background of $27 \pm 5$ (stat.) \pm 2 (syst.): a $1.5 \sigma$ excess. The resulting fit to an oscillation model is shown in figure 5, compared to the limit from Chooz [7]. 


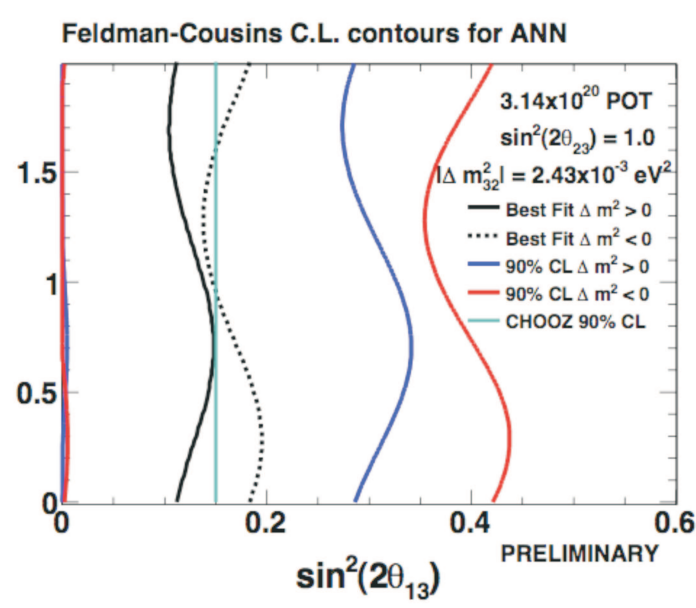

Figure 5: The MINOS 90\% allowed regions for $\sin ^{2}\left(2 \theta_{13}\right)$ for the normal (blue) and inverted (red) mass hierarchies. Also shown are the MINOS best fit value (black) and the Chooz 90\% limit (grey).

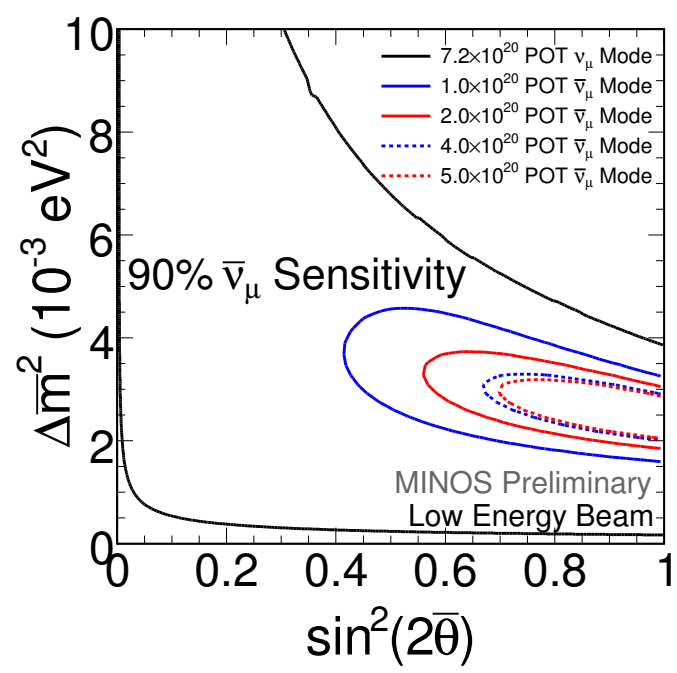

Figure 6: The MINOS sensitivity to $\bar{v}_{\mu}$ oscillation parameters for the $7 \% \bar{v}_{\mu}$ background in the NuMI $v_{\mu}$ beam (black) and for dedicated $\bar{v}_{\mu}$ running (blue and red). One year of $\bar{v}_{\mu}$ running corresponds to $\sim 2.0 \times 10^{20}$ protons on target (POT).

\section{The Future}

The analyses presented above have used a dataset of $3 \times 10^{20}$ protons on target (POT). As of the June 2009 shutdown, MINOS has recorded $7 \times 10^{20}$ POT. Work is underway to update all these analyses with this doubled dataset; results are expected by the summer of 2010 .

In September 2010, the current in the NuMI focusing horns will be reversed, producing a $\bar{v}_{\mu}$ beam. This will allow MINOS to obtain a greatly enhanced $\bar{v}_{\mu}$ sample, concentrated around the expected oscillation maximum. With this, MINOS will make the first ever precision measurement of the atmospheric neutrino oscillation parameters in the atmospheric regime, being able to precisely test CPT conservation in the neutrino sector. After one year of data-taking $\left(\sim 2 \times 10^{20}\right.$ POT), $\bar{v}_{\mu}$ oscillations can be observed at the $5 \sigma$ level. Figure 6 shows the MINOS sensitivity to $\bar{v}_{\mu}$ oscillations, compared to the current sensitivity using the $\bar{v}_{\mu}$ background in the NuMI $v_{\mu}$ beam.

\section{References}

[1] D.G. Michael et al., Nucl. Instr. and Meth. A 596 (2008) 190.

[2] P. Adamson et al., Phys. Rev. Lett. 101, 131802 (2008).

[3] V. Barger et al., Phys. Rev. Lett. 82, 2640 (1999).

[4] G.L. Fogli et al., Phys. Rev. D 67, 093006 (2003).

[5] M.C. Gonzalez-Garcia and M. Maltoni, Phys. Rept. 460, 1 (2008).

[6] P.A. Adamson et al., Phys. Rev. Lett. 101, 221804 (2008).

[7] M. Apollonio et al., Eur. Phys. J. C 27, 331 (2003). 